\title{
Biodegradation of Polyvinyl Alcohol by Thai Indigenous Mixed Microbial Culture
}

\section{Duangkhaetita KANJANASOPA, ${ }^{1, *}$, Benchamaporn PIMPA ${ }^{2}$, Suraphon THITITHANAKUL ${ }^{1}$ and Suwaluk WISUNTHORN ${ }^{3}$}

\author{
${ }^{1}$ Agricultural Science and Technology Program, Faculty of Science and Industrial Technology, \\ Prince of Songkla University, Surat Thani Campus, Surat Thani 84000, Thailand \\ ${ }^{2}$ Food Technology Program, Faculty of Science and Industrial Technology, Prince of Songkla University, \\ Surat Thani Campus, Surat Thani 84000, Thailand \\ ${ }^{3}$ Rubber Technology Program, Faculty of Science and Industrial Technology, \\ Prince of Songkla University, Surat Thani Campus, Surat Thani 84000, Thailand
}

('Corresponding author’s e-mail: daungkhae.k@psu.ac.th)

Received: 26 October 2018, Revised: 4 July 2019, Accepted: 9 August 2019

\begin{abstract}
PVA is biodegradable plastic and a water-soluble synthetic polymer that plays a significant role in industry. A large amount of PVA in wastewater causes heavy environmental pollution in terms of accumulation, disposal, and long-term degradation; therefore it must be removed from wastewater before the water is discharged. In this study, NS3 mixed microbial culture, capable of completely degrading 5 g. $\mathrm{L}^{-1}$ polyvinyl alcohol (PVA), was isolated from landfill soil using the enrichment culture method. It completely degraded PVA at an initial concentration in the range $1-5$ g. $\mathrm{L}^{-1}$ over $5-20$ days of incubation with continuous shaking at $30{ }^{\circ} \mathrm{C}$. Moreover, mixed microbial cultures were found to remove PVA at a high range concentration of $10-25$ g. $\mathrm{L}^{-1}$. Urea and glucose added to the medium inhibited PVA degradation by increasing the $\mathrm{pH}$ to a strongly alkaline level, which would cause cell viability and enzyme stability. The FT-IR spectra and SEM imaging revealed the mechanisms and the physical degradation of PVA films, respectively. PVA uptake in bacterial cells produced a dent in the cell surface, which represented the consumption of PVA by bacterial cell. The PVA-degrading mixed microbial culture is the first reported in Thailand and can be beneficial in PVA wastewater treatment.
\end{abstract}

Keywords: Biodegradation, Polyvinyl alcohol, PVA degrading microorganism, Mixed microbial cultures

\section{Introduction}

Polyvinyl alcohol, PVA, is a well-known synthetic polymer that is attracting interest for the production of biodegradable plastic materials. The main chains of the vinyl polymer are joined only by carbon-carbon linkages, similar to typical plastics such as polyethylene, polypropylene, and polystyrene. The major uses of PVA include textiles, paper coating, warp sizing, adhesives, and films. PVA is a watersoluble polymer. The large amounts of the material used and discarded have become a significant waste problem. Among the vinyl polymers produced industrially, PVA is the only one known to be mineralized by microorganisms, but its natural biodegradation rate is slow [1]. Many studies have screened for isolated PVA degrading microorganisms. Single species, symbiotic cultures, and mixed microbial cultures have been reported. Most single species that degrade PVA require the addition of exogenous pyrroloquinoline quinine (PQQ) to the medium. PQQ is a cofactor for PQQ-dependent PVA dehydrogenase (PVADH), which catalyzes the initial oxidation step in PVA degradation [1]; for example, Pseudomonas spp. [2], Alcaligenes faecalis [3], and Sphingopyxis sp. PVA3 [4]. In symbiotic system 
http://wjst.wu.ac.th

cultures, one symbiont usually provides PQQ or other factors for complete PVA degradation. A cooperative function of two strains may be required for PVA degradation, such as: Pseudomonas sp. VM15C, Bacillus megaterium, Sphingomonas sp. SA3, and Cardiobacterium sp. SB68 (SB68), for symbionts: Pseudomonas putida VM15A, bacterial strain PN19, SA2, and an unidentified bacterium (SB69), respectively [5-8]. In some mixed microbial cultures, synergistic effects exist between strains $[9,10]$. Several different enzyme systems for the degradation of PVA's main chain have been reported [11].

As the number of PVA-degrading microorganisms is limited, and many of those that have been identified do not seem to be applicable, it is still necessary to search for novel microorganisms or enzymes with good degradation capabilities. Novel microorganisms that degrade PVA faster or more readily (i.e., without symbiotic or supplemental compounds) may be used to enhance the degradation of PVA in wastewater or soil. This would also raise the status of PVA as a biodegradable polymer. In this study, PVA-degrading microorganisms were initially screened and identified from soils contaminated with PVA in Thailand. We focus on Thai indigenous organisms that are comparatively easier to introduce and practice in Thailand. The various conditions of PVA degradation were also experimentally determined. Finally, the PVA degradation by the mixed culture was characterized both by FT-IR and by a scanning electron microscope.

\section{Materials and methods}

\section{Chemicals and medium}

Commercial PVA powders (PVA 117K, PVA 205MB, and PVA 217SB) were purchased from the Kuraray Company (Japan). The media and chemicals were purchased from the Hi-media, Fluka, Merck, and Sigma companies. Mineral salt medium (MM) containing $0.5 \%(\mathrm{w} / \mathrm{v})$ PVA $117 \mathrm{~K}$ was the screening medium for PVA degrading microorganisms. One liter of MM medium contains $1 \mathrm{~g}$ of $\left(\mathrm{NH}_{4}\right)_{2} \mathrm{HPO}_{4}, 1.6$ $\mathrm{g}$ of $\mathrm{K}_{2} \mathrm{HPO}_{4}, 0.2 \mathrm{~g}$ of $\mathrm{MgSO}_{4} .7 \mathrm{H}_{2} \mathrm{O}$ and $0.2 \mathrm{~g}$ of $\mathrm{KH}_{2} \mathrm{PO}_{4}, 0.5 \mathrm{~g}$ of peptone, $0.5 \mathrm{~g}$ of yeast extract, $1 \mathrm{~mL}$ of trace element solution $\left(0.5 \mathrm{mg}\right.$ of $\mathrm{Na}_{2} \mathrm{MoO}_{4} \cdot 2 \mathrm{H}_{2} \mathrm{O}, 0.5 \mathrm{mg}$ of $\mathrm{MnSO}_{4}, 10 \mathrm{mg}$ of $\mathrm{NaCl}$ and $20 \mathrm{mg}$ of $\mathrm{CaCl}_{2} \cdot 2 \mathrm{H}_{2} \mathrm{O}$ ), $1 \mathrm{~mL}$ of vitamin solution (Inositol $0.2 \mathrm{mg}$, Pyridoxine $0.4 \mathrm{mg}$, Thiamine $0.4 \mathrm{mg}$ and Nicotinic acid $0.2 \mathrm{mg}$ ), and $10 \mathrm{mg}$ of $\mathrm{FeSO}_{4} \cdot 7 \mathrm{H}_{2} \mathrm{O}$. To add $0.5 \%(\mathrm{w} / \mathrm{v}) \mathrm{PVA}$ to the medium, $100 \mathrm{~mL}$ of a $5 \%(\mathrm{w} / \mathrm{v})$ PVA $117 \mathrm{~K}$ solution was used. The mixture of ingredients was then diluted with a one-liter volume of distilled water, so that the PVA concentration was $0.5 \%$. The $5 \%(\mathrm{w} / \mathrm{v})$ PVA solutions were prepared by dissolving $5 \mathrm{~g}$ of PVA powder in $100 \mathrm{~mL}$ distilled water and autoclaving. Fifteen grams of Bacto-agar was used to create a solid medium. The basic medium (BM) used in this study was composed of $1 \mathrm{~g}$ of $\mathrm{NH}_{4} \mathrm{NO}_{3}, 1 \mathrm{~g}$ of yeast extract, $1.6 \mathrm{~g}$ of $\mathrm{K}_{2} \mathrm{HPO}_{4}, 0.05 \mathrm{~g}$ of $\mathrm{MgSO}_{4} . \mathrm{H}_{2} \mathrm{O}, 0.05 \mathrm{~g}$ of $\mathrm{CaCl}_{2}, 0.02 \mathrm{~g}$ of $\mathrm{FeSO}_{4} .7 \mathrm{H}_{2} \mathrm{O}$, and $0.02 \mathrm{~g}$ of $\mathrm{NaCl}$ per liter final volume [12].

\section{Screening for PVA degrading bacteria}

Sixteen soil samples were collected randomly from landfills contaminated with PVA. Approximately one hundred grams of soil from 1-2 cm below the surface was sampled. The enrichment culture method was modified from [12]; $5 \mathrm{~g}$ of each sample were added into $45 \mathrm{~mL}$ normal saline and shaken with a shaker for $1 \mathrm{~h}$. Then, $2 \mathrm{~mL}$ of this solution was inoculated into a $250 \mathrm{~mL}$ flask containing $50 \mathrm{~mL}$ of the screening medium and cultured at $30^{\circ} \mathrm{C}$ on a rotary shaker at $150 \mathrm{rpm} . \mathrm{min}^{-1}$ for 7 days. For the enrichment culture, the mixed microbial culture was further inoculated to new screening medium twice. A soil sample (actually the mixed microbial culture from it) that could completely degrade 5 g.L $\mathrm{L}^{-1}$ of PVA117K was chosen for further study. Five different bacterial strains were isolated from the mixed culture by the dilution method and were named as strain No. 1 - 5 .

\section{Flask culture conditions and PVA degradation patterns}

A starter culture was prepared by re-culturing the mixed microbial culture, isolated strain, or the mixture of isolated strain (a combination of equal volume for each isolated bacterial culture) in PVA broth. For this, $2 \%(\mathrm{v} / \mathrm{v})$ of the NS3 mixed microbial culture, a single isolated culture, or the mixture of isolated strain was inoculated to $50 \mathrm{~mL}$ of $0.5 \%$ PVA medium. The culturing was carried out on a rotary 
shaker at $150 \mathrm{rpm} \cdot \mathrm{min}^{-1}$ and at $30{ }^{\circ} \mathrm{C}$ until the PVA was consumed. PVA degradation patterns from the mixed microbial culture were studied at different PVAs (PVA 205MB, PVA 217SB, and PVA117K), medium types, temperatures, shaking speeds, PVA concentrations $\left(1,5,10\right.$, or 25 g.L. $L^{-1}$, and supplementations of urea and/or glucose $\left(0\right.$ or $\left.1 \mathrm{~g} . \mathrm{L}^{-1}\right)$ in the medium. The amount of PVA in the culture medium was measured by iodometry according to the Finley method [13].

\section{Analysis of PVA concentration}

Quantitative determination of the residual PVA concentration was carried out by Finley's method [13]. The method is based on the blue color produced by the reaction of PVA with iodine in the presence of boric acid. The details of this determination are described as follows: $1 \mathrm{~mL}$ of cell culture was taken and centrifuged to precipitate the cells. The supernatant was collected and diluted by distilled water to an appropriate concentration, $0.5 \mathrm{~mL}$ of the diluted supernatant was mixed with $0.75 \mathrm{~mL}$ of $4 \%(\mathrm{w} / \mathrm{v})$ boric acid, and $0.15 \mathrm{~mL}$ of $\mathrm{KI}_{-} \mathrm{I}_{2}$ solution was added. The mixture was left at room temperature for $20 \mathrm{~min}$, and then $2 \mathrm{~mL}$ of distilled water was added. The optical absorbance of the reaction mixture was detected at $660 \mathrm{~nm}$. The optical values were compared to a PVA standard curve to determine the concentration of residual PVA in the medium. A blank culture was used as the reference during the tests. The percentage of degradation was determined based on the difference of the concentration of initial PVA $\left(\mathrm{A}_{0}\right)$ and residual PVA $\left(\mathrm{A}_{\text {res }}\right)$ divided by initial PVA $\left(\mathrm{A}_{0}\right)$ and multiplied by 100 . The standard curve was prepared for a PVA range from 0.01 to $0.1 \mathrm{mg}$.

\section{Fourier transform infrared spectrophotometry}

PVA degradation by the mixed microbial culture was assessed by FT-IR. Thirty milliliters of the mixed microbial culture was collected at 10 and 30 days. Culture supernatant was collected after centrifuging at $10,000 \mathrm{rpm} \cdot \mathrm{min}^{-1}$ for $10 \mathrm{~min}$ at $4{ }^{\circ} \mathrm{C}$. Culture supernatants were freeze dried and used for the FT-IR analyses. Powder form PVA $117 \mathrm{~K}$ was used as the control. The FT-IR spectra were recorded using a Fourier Transform Infrared Spectrophotometer model EQUINOX55, Bruker, with a KBr disk as the blank, scanning from 4,000 to $400 \mathrm{~cm}^{-1}$ at a resolution of $4 \mathrm{~cm}^{-1}$.

\section{Morphological observations}

A PVA film was aseptically prepared by pouring $5 \%(\mathrm{w} / \mathrm{v})$ PVA117K solution in a petri dish. It was dried and cut into small $3 \times 4 \mathrm{~cm}$ samples. Fifty milliliters of medium containing a PVA film sample was inoculated with the NS3 mixed microbial culture and shaken at $30{ }^{\circ} \mathrm{C}$ and $150 \mathrm{rpm} . \mathrm{min}^{-1}$ for 40 days. The PVA film samples were collected, dried, and vacuum coated with gold for imaging with a scanning electron microscope (SEM) (Quanta400, FEI). The degradation was assessed from the images. The controlled PVA film sample treated without inoculum was assessed similarly.

\section{Results and discussion}

\section{Screening of PVA degrading microorganisms}

Sixteen soil samples were screened for PVA degrading bacteria using PVA enriched medium cultures. Only one soil sample, namely NS3, totally degraded PVA initially at 5 g. $\mathrm{L}^{-1}$ within twenty days of culture (Figure 1a). It was collected from a landfill at Ban Nasan, Surat Thani province, which was contaminated via PVA composting. Five strains (No. 1 - 5) were isolated from the NS3 mixed microbial culture via the dilution method. Cultivation of these five bacteria cultures together or individually in a PVA medium no longer showed complete degradation ability. This might be due to the loss of some strain during isolation, so that symbiotic or synergistic conditions were lost. During the cultivation of the NS3 mixed microbial culture in PVA medium, bacterial cells grew rapidly where a large amount of cell flocculation was produced within 7 days. We detected PVA reduction after cell coagulation appeared, while the mixture of isolated strains only grew in PVA medium and neither cell coagulation nor PVA degradation occurred. In this study we observed large amounts of mixed bacterial coagulation, which could predict the PVA elimination in broth culture. 
http://wjst.wu.ac.th
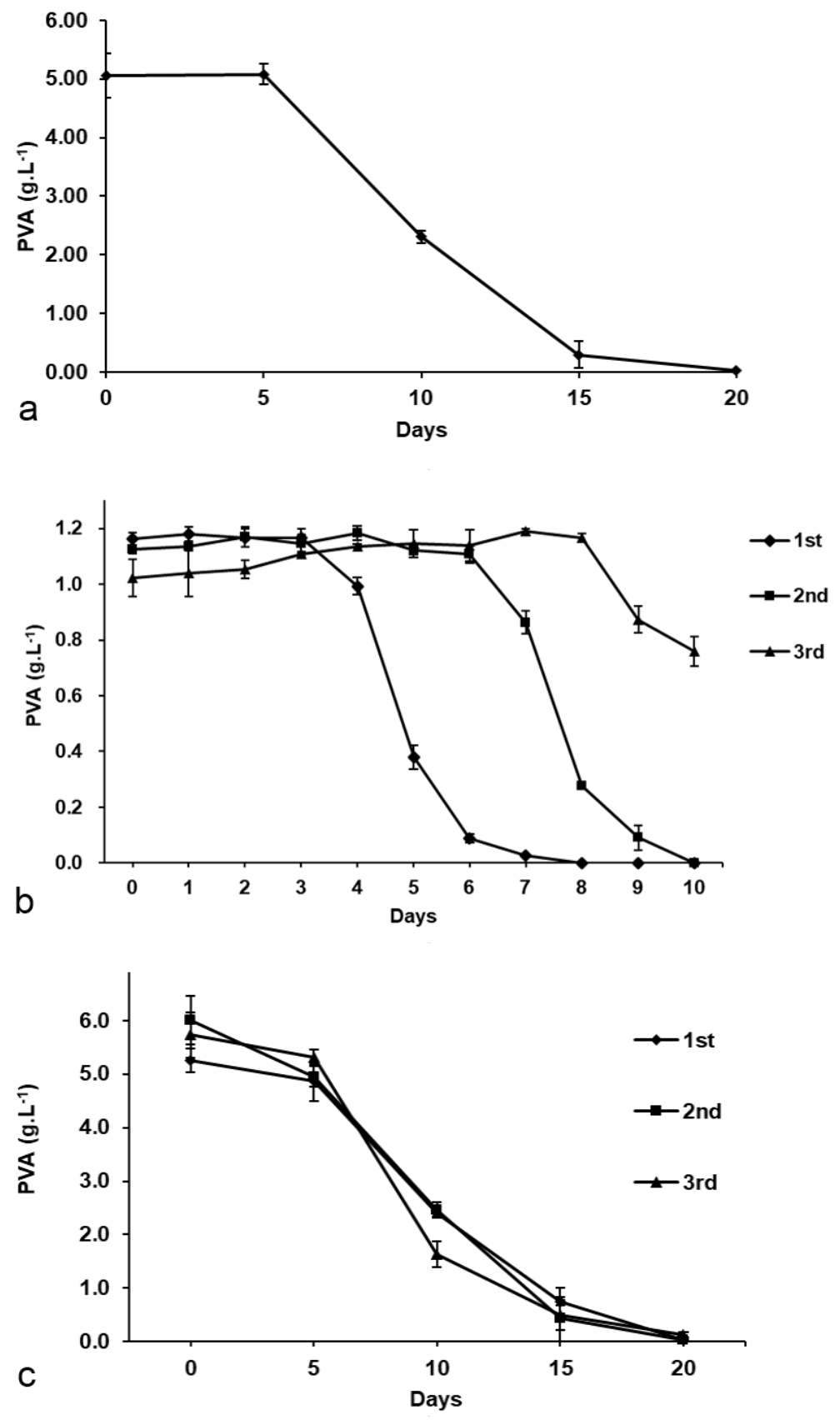

Figure 1 PVA degradation by NS3 mixed microbial culture cultivated in a PVA medium and the effects of PVA concentration on PVA degradation over time. a) The time profile of residual PVA in broth culture with initially $0.5 \%(\mathrm{w} / \mathrm{v})$ PVA. b) and c) The mixed microbial cultures were cultivated at $0.1 \%(\mathrm{w} / \mathrm{v})$ and $0.5 \%(\mathrm{w} / \mathrm{v})$ of PVA with shaking at $30{ }^{\circ} \mathrm{C}$ and $150 \mathrm{rpm} \cdot \mathrm{min}^{-1}$. The symbols indicate the cycle number of re-cultivation as first $\left(1^{\text {st }}\right)$, second $\left(2^{\text {nd }}\right)$, and third $\left(3^{\text {rd }}\right)$. 
The PVA degradation ability might change during cycles of culturing, depending on the PVA concentration. The ability to degrade PVA was reduced when the mixed microbial culture was recultivated in $0.1 \%(\mathrm{w} / \mathrm{v})$ PVA (Figure 1b), while its PVA degrading ability was maintained when PVA concentration was $0.5 \%(\mathrm{w} / \mathrm{v})$ (Figure 1c). Notably, cultivation of the mixed microbial culture with low PVA concentrations reduced the amount of cell coagulation. It is reasonable that PVA degradation may have an initiation step dependent on the presence and amount of a certain strain in this mixed microbial culture. Some research proposed that the PVA degrading process with mixed microbial culture [12] strain grows in the beginning of cultivation and cuts PVA chains with a high degree of polymerization. Both extracellular and membrane-oriented PVA degrading enzymes can be produced to cut PVA into smaller molecules. As the degree of polymerization of PVA decreases, other microorganisms can utilize the smaller PVA fragments and thrive. Eventually, small fragments emerge to enter into some microorganisms that produce intracellular enzymes for further degradation of the intermediates. Thus, the microorganisms in a NS3 mixed microbial culture may sequentially degrade PVA in a synergistic fashion.

\section{Degradation of the different PVA samples}

The behavior of mixed microbial culture on biodegradation of the different PVAs was studied in flask cultures. The types of PVA were PVA 117K, PVA 205MB, and PVA 217SB, and they showed similar time profiles of degradation (Figure 2a). This indicates that the mixed microbial culture degraded PVAs over a wide range of degrees of hydrolysis, as PVA $117 \mathrm{~K}$ has a high degree of hydrolysis $(98-99$ $\%$ mol), different from PVA 205MB and PVA 217SB (87 - $89 \%$ mol). Fortunately, the NS3 mixed culture degraded all these PVA types, which are routinely used in adhesives, paper coatings, and textile products, suggesting that it has potential for eliminating PVA contamination in the environment. Nevertheless, the mixed microbial culture isolated from the Pacific Textile Factory in China preferred degrading PVA with low polymerization over types with high polymerization [12]. This might depend on the bacterial consortium in a mixed microbial culture.

\section{Culture conditions for PVA degradation}

The choice of medium for PVA degradation was examined. The BM gave $97 \%$ of polymer degrading, while MM gave $86 \%$ after 15 days of incubation. The $\mathrm{pH}$ of both media was mostly stable and neutral throughout cultivation (Figure 2b). Due to its simpler composition and easier preparation, the $\mathrm{BM}$ was chosen for further experiments. A comparison of the degradation time profiles at 30 and $35{ }^{\circ} \mathrm{C}$ indicated similar completion times, but $30^{\circ} \mathrm{C}$ showed higher intermediate rates (Figure 2c). Moreover, lower culture temperatures under $30{ }^{\circ} \mathrm{C}$ retarded PVA biodegradation (data not shown). Continuous shaking increased PVA degradation, whereas no or limited shaking failed at the early period of culturing for polymer elimination. However, PVA degradation at this insufficient aeration was revealed after prolonged culturing over 15 days (Figure 2d). This is possibly due to aeration by the enhancing growth of the first active strains in the mixed culture, producing essential growth factors for the other strains that grew relatively slowly to secrete the enzymes for starting actual PVA degradation [14]. The proposition is that early stages of cultivation showed that some strains grew without PVA degradation. In addition, PVA degradation has been detected with prolonged cultivation past five days, which was corroborated by the current experiments. Most PVA degrading microorganisms have been identified as aerobic bacteria belonging to Proteobacteria, Firmicute, Fungi, and Actinomycete [5]. An anaerobic-aerobic bioreactor model showed that the dominant bacteria degrading PVA were Proteobacteria [16]. This indicated that Proteobacteria could grow under both anaerobic and aerobic conditions and were responsible for PVA degradation. This phylum has been demonstrated to dominate printing and dyeing wastewater [17] and showed abundant bacteria species in the mixed culture on PVA degradation processed. [12].

PVA degradation by a mixed microbial culture was investigated at different initial PVA

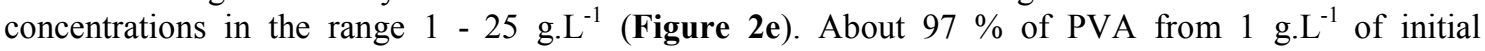
concentration was removed within 5 cultivation days. The mixed microbial culture removed the polymer by about 90,80 , and $50 \%$ within 20 cultivation days when the initial PVA concentration was increased to 5,10 , and 25 g. $\mathrm{L}^{-1}$, respectively. This reveals that the NS3 mixed microbial culture has potential for the 
degradation of PVA at high concentrations. Prior reports have found that high PVA concentration inhibited degradation by a mixed microbial culture [18]; however, the mixed microbial culture in this experiment still started PVA degradation, even though PVA concentration was increased to 25 g. $\mathrm{L}^{-1}$. All these results suggest that superior biodegradation depends upon the composition of a microbial community, and that it is synergistic. As mentioned, NS3 mixed microbial may possibly have an effective application in textile wastewater treatment under limiting aeration and PVA concentration load.
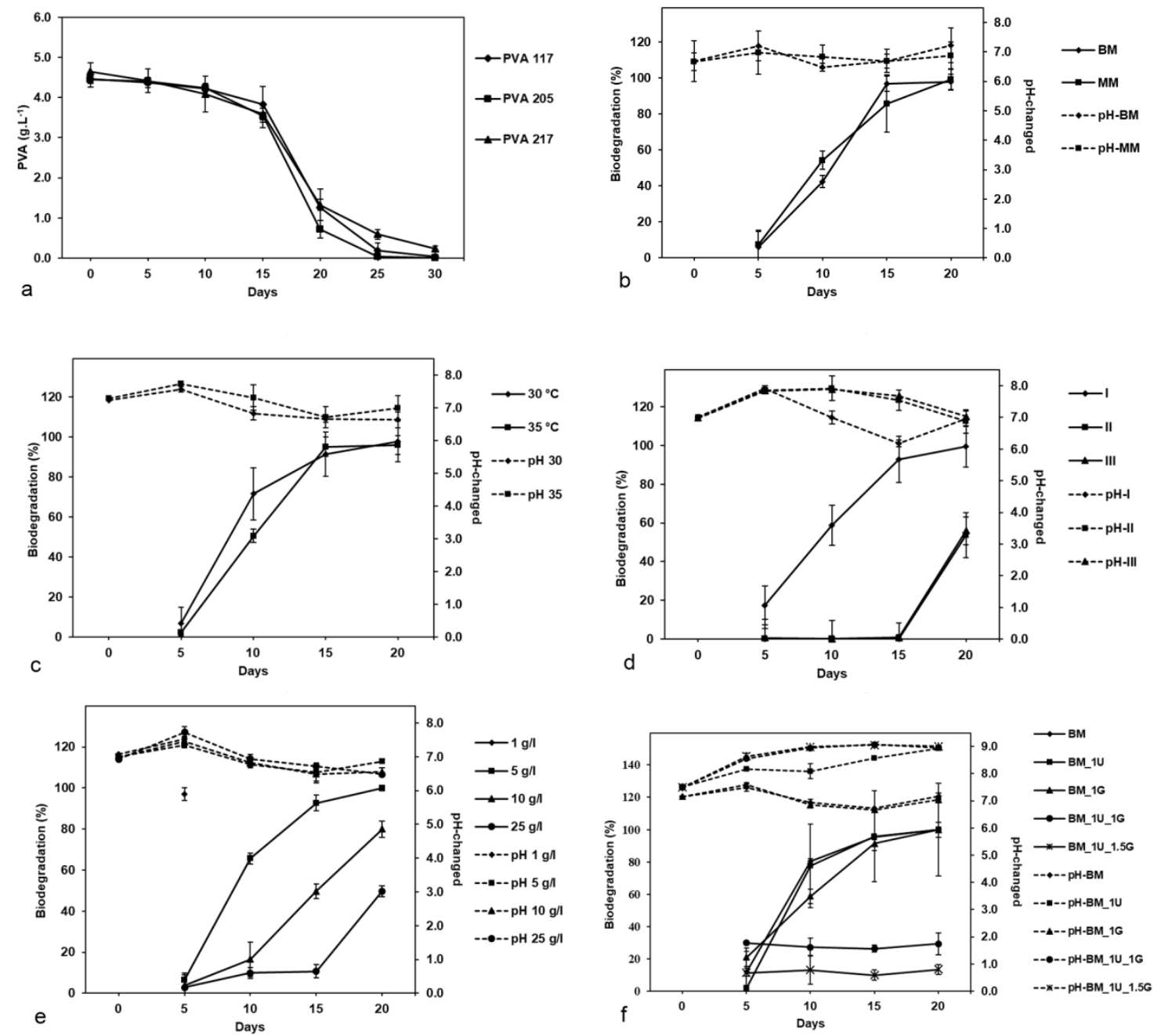

Figure 2 Factors affected PVA degradation by the NS3 mixed microbial culture. a) Degradation of different types of PVA. b) Comparison of medium types MM and BM. Effects of choices of c) culture temperature at 30 and $35^{\circ} \mathrm{C}$, d) shaking speed (I) continuous shaking at $150 \mathrm{rpm} . \mathrm{min}^{-1}$; (II) shaking for 30 min per day at $150 \mathrm{rpm} . \mathrm{min}^{-1}$; or (III) without shaking, e) PVA concentration, and f) supplementation with urea and/or glucose in medium. All studies used $0.5 \%(\mathrm{w} / \mathrm{v})$ PVA as the initial concentration, except for e. 
According to prior reports, the degradation of PVA can depend on the medium composition. Inorganic nitrogen sources such as $\mathrm{NaNO}_{3}, \mathrm{NH}_{4} \mathrm{NO}_{3},\left(\mathrm{NH}_{4}\right)_{2} \mathrm{SO}_{4}$, and urea have enhanced PVA degradation more than organic nitrogen sources, such as yeast extract, peptone, or beef extract [12]. The ratio of carbon sources to nitrogen sources and the $\mathrm{pH}$ of the medium influence PVA degradation. We tried supplementing carbon and nitrogen in the medium to increase PVA degradation. Urea as an inorganic nitrogen source was added in the basic medium at $1 \mathrm{~g} . \mathrm{L}^{-1}$ to help evaluate PVA degradation. The polymer removal pattern was similar to that without supplemented urea, but during the cultivation it increased the $\mathrm{pH}$ to alkaline levels 7.5 - 8.5. Urea did not promote PVA degradation because sufficient nitrogen was already available in the medium (Figure 2f). Glucose supplemented at an initial concentration 1 g.L $\mathrm{L}^{-1}$ in the medium gave 2-fold higher polymer degradation, changing it from $11 \%$ to 20 $\%$ at 5 days of cultivation. It was preferentially utilized by bacterial consortiums for growth and consequently increased initial biodegradation. This is called the "priming effect", when glucose stimulates PVA mineralization [9]. However, adding glucose to the medium decreases PVA degradation over long periods of time. The equal combination of urea and glucose at an initial concentration 1 g. $\mathrm{L}^{-1}$ promoted PVA degradation to $30 \%$ over the initial culture period, and then completely inhibited it in prolonged culturing (Figure 2f). Moreover, the higher the glucose concentration, the stronger the inhibition observed. The $\mathrm{pH}$ of culture media containing urea and glucose varied within $7.5-9.05$ at the end of incubation. Adding urea and glucose increased the $\mathrm{pH}$ to strongly alkaline levels, causing the demise of microorganisms and the instability of PVA degrading enzymes. The $\mathrm{pH}$ influences all biological processes, including growth and PVA degradation activities of the microorganisms. The enzymes secreted by mixed Bacillus sp. and Curtobacterium sp. actively degrade PVA in an alkaline solution with initial pH 8 [9]. In another case, the enzyme activity of PVA degrading bacteria decreased when the $\mathrm{pH}$ of the medium was not held at the optimally neutral $(\mathrm{pH} 7)$ level [19]. The optimal $\mathrm{pH}$ value for PVA degradation by a mixed culture isolated from paper mill treatment was $\mathrm{pH} 8$, showing the shortest period for complete degradation [18]. All these data corroborate this current study, in that PVA removal was best when $\mathrm{pH}$ was about $7-8$, which is suitable for bacterial growth and the stability and function of PVA degrading enzymes.

\section{Investigation of PVA biodegradation by FTIR}

PVA is a polymer with a carbon chain backbone and hydroxyl groups attached to methane carbon [20]. Kawai and $\mathrm{Hu}$ [11] reported that the extensive published studies have established a two-step process in the biodegradation of PVA, first by oxidation of two hydroxyl groups leading to $\beta$-diketone structures or oxidation of one hydroxyl group obtaining monoketone structures, and then second by either hydrolysis of $\beta$-diketone structures of oxidized PVA (oxiPVA) by a $\beta$-diketone hydrolase (oxiPVA hydrolase) or aldolase reaction involving the monoketone structures of oxiPVA. To assess the biodegradation of PVA in our experiments, FTIR spectra were measured for biodegradation over 30 days. Two spectra are shown in Figure 3 (native PVA (a) and PVA degraded for 30 days (b)). The spectrum of the native PVA was similar in its coarse features to the spectra of PVA biodegraded for the 30 day periods. The peak in the region $3,600-2,600 \mathrm{~cm}^{-1}$ is related to stretching vibrations of OH group [21]. It is attributed here to the carboxyl groups presented in all samples. An absorption band at $1,714 \mathrm{~cm}^{-1}$ was attributed to the carboxyl group in PVA, and was due to the stretching of $\mathrm{C}=\mathrm{O}$ and $\mathrm{C}-\mathrm{O}$. However, it is missing from PVA biodegraded for 30 days. Additional absorption peaks at 1,651 and 1,404 $\mathrm{cm}^{-1}$ were observed for 30 days, which were identified as a $\beta$-diketone structure and methyl formed by the scission of several carbon bonds, respectively [9].

The morphologies of PVA and degraded PVA were observed under SEM (Figure 4). The microbial populations on the PVA films held in the medium for 40 days were observed (Figures 4 b - 4d). Some bacteria appeared on the film surface and penetrated into the PVA matrix. In addition, a dent appeared in the cell surface when culturing for 40 days (Figure 4d). The cell surface structural change was similar with Sphingopyxis sp. strain 113P3 cultivated for 2 days on PVA medium [22] and Sphingomonas sp. A1 after exposure to alginate [23]. The dent appearing on bacteria seemed to be playing a role similar to that of them. The cell surface structure change is induced by PVA, and a dent in the cell surface seem to be 
related to the uptake of PVA. These morphologies confirm the degradation of PVA using mixed microbial culture.

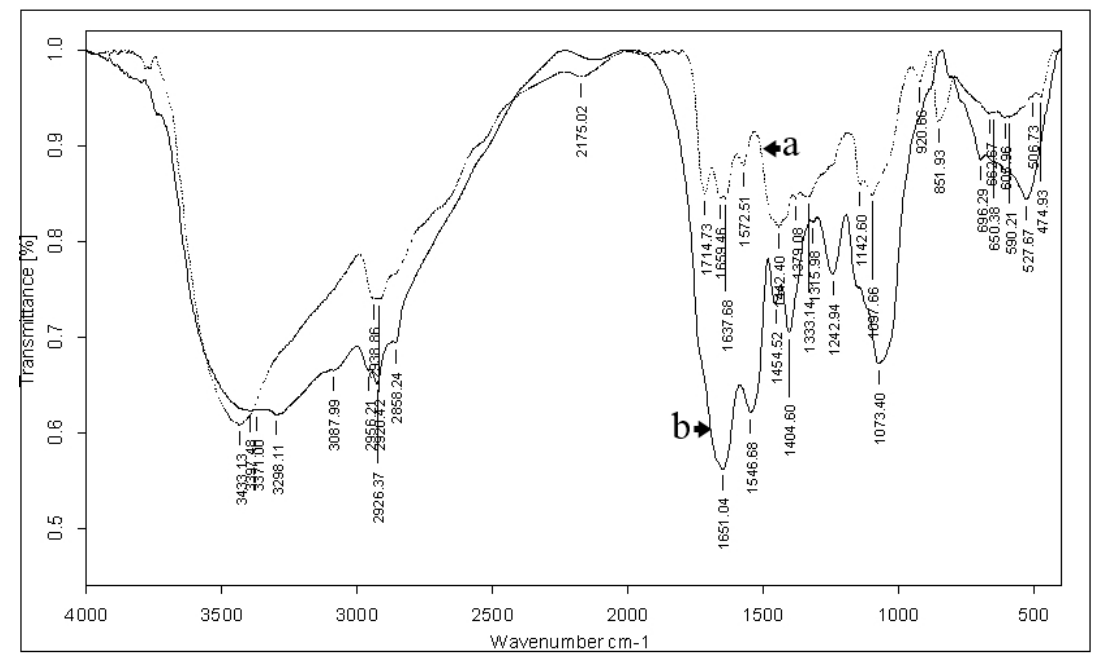

Figure 3 FTIR spectra of native PVA (a) and PVA degraded for 30 days (b).

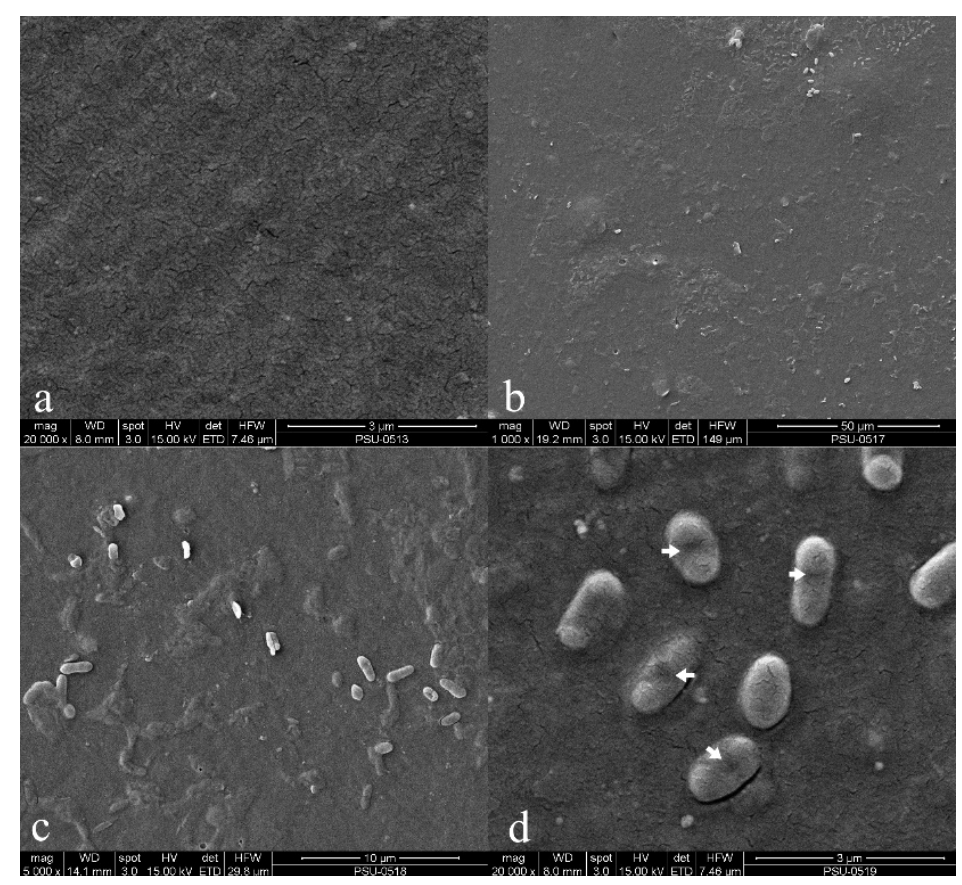

Figure 4 SEM images of PVA films incubated in the medium for 40 days. PVA film incubated without inoculation of bacteria was used as the control, shown in a) at 20,000×. b, c, d) The NS3 mixed bacteria are visible at the magnifications of $1,000 \times, 5,000 \times$, and 20,000×, respectively. Arrows indicated a dent in the bacterial cell surface. 


\section{Conclusions}

In conclusion, NS3, a mixed microbial culture, degraded PVA types with both high and low degrees of hydrolysis. PVA degradation occurred best in cultures at $30{ }^{\circ} \mathrm{C}$ with shaking at $150 \mathrm{rpm} . \mathrm{min}^{-1}$. The NS3 degraded PVA at concentrations from 1 to $25 \mathrm{~g} . \mathrm{L}^{-1}$ but, at the high end of this range, the degradation rates were comparatively low. A combination of urea and glucose repressed PVA assimilation because the culture turning alkaline could affect the viability of microorganisms and the stability of PVA degrading enzymes. FT-IR analyses of the PVA samples with and without degradation were assessed to gain insights into the degradation mechanisms and intermediate products. SEM imaging showed the physical degradation of PVA films and ultrastructural change in the cell surface of bacteria relating to the PVA uptake into the cell.

\section{Acknowledgements}

The authors acknowledge financial support from the Prince of Songkla University No. TAM50200200246S and the International Foundation for Science (IFS). The authors thank Assoc. Prof. Dr. Seppo Karrila for helpful comments and suggestions.

\section{References}

[1] M Shimao. Biodegradation of plastics. Curr. Opin. Biotechnol. 2001; 2, 242-7.

[2] K Sakai, M Fukuba, Y Hasui, K Moriyoshi, T Ohmoto, T Fujita and T Ohe. Purification and characterization of an esterase involved in poly (vinyl alcohol) degradation by Pseudomonas vesicularis PD. Biosci. Biotechnol. Biochem.1998; 62, 2000-7.

[3] S Mutsumura, N Tomizawa, A Toki, K Nishikawa and K Toshima. Effect of molecular-weight and stereo-regularity on degradation of poly (vinyl alcohol) by Alcaligenes faecalis. Biotechnol. Lett. 1994; 16, 1205-10.

[4] A Yamatsu, R Matsumi, H Atomi and T Imanaka. Isolation and characterization of a novel poly (vinyl alcohol)-degrading bacterium", Sphingopyxis sp. PVA3. Appl. Microbiol. Biotechnol. 2006; 72, 804-11.

[5] M Shimao, K Ninomiya, O Kuno, N Kato and C Sakazawa. Existence of novel enzyme, pyrroloquinoline quinine-dependent polyvinyl alcohol dehydrogenase, in a bacterial symbiont, Pseudomonas sp. strain VM15C. Appl. Environ. Microbiol. 1986; 5, 268-75.

[6] T Mori, M Sakimoto, T Kagi and T Sakai. Isolation and characterization of a strain of Bacillus megaterium that degrades poly (vinyl alcohol). Biosci. Biotechnol. Biochem. 1996; 60, 330-2.

[7] BC Kim, CK Sohn, SK Lim, JW Lee and W Park. Degradation of polyvinyl alcohol by Sphingomonas sp. SA3 and its symbiote. J. Ind. Microbiol. Biotechnol. 2003; 30, 70-4.

[8] JA Lee and MN Kim. Isolation of new and potent poly (vinyl alcohol)-degrading strains and their degradation activity. Polym. Degradation Stab. 2003; 81, 303-8.

[9] $\mathrm{HZ}$ Zhang. Influence of $\mathrm{pH}$ and $\mathrm{C} / \mathrm{N}$ ratio on poly (vinyl alcohol) biodegradation in mixed bacterial culture. J. Polym. Environ. 2009; 17, 286-90.

[10] B Tang, X Liao, D Zhang, M Li, R Li, K Yun, G Du and J Chen. Enhanced production of poly (vinyl alcohol)-degrading enzymes by mixed microbial culture using 1,4-butanediol and designed fermentation strategies. Polym. Degradation Stab. 2010; 95, 557-63.

[11] F Kawai and XP Hu. Biochemistry of microbial polyvinyl alcohol degradation. Appl. Microbial. Biotechnol. 2009; 84, 227-37.

[12] J Chen, Y Zhang, GC Du, ZZ Hau and Y Zhu. Biodegradation of polyvinyl alcohol by a mixed microbial culture. Enzyme Microb. Tech. 2007; 40, 1686-91.

[13] JH Finley. Spectrophotometric determination of polyvinyl alcohol in paper coatings. Anal. Chem. 1961; 33, 1925-7.

[14] T Vaclavkova, J Ruzicka, M Julinova, R Vicha and M Koutny. Novel aspects of symbiotic (polyvinyl alcohol) biodegradation. Appl. Microbiol. Biotechnol. 2007; 76, 911-7. 
[15] D Qian, G Du and J Chen. Isolation and culture characterization of a new polyvinyl alcoholdegrading strain: Penicillium sp. WSH02-21. World J. Microbe. Biot. 2004; 20, 587-91.

[16] J Huang, S Yang and S Zhang. Performance and diversity of polyvinyl alcohol-degrading bacteria under aerobic and anaerobic conditions. Biotechnol. Lett. 2016; 38, 1875-80.

[17] Q Yang, W Zhang, H Zhang, Y Li and C Li. Wastewater treatment by alkali bacteria and dynamics of microbial communities in two bioreactors. Bioresour. Tech. 2011; 102, 3790-8.

[18] D Cetin and S Citak. Degradation of polyvinyl alcohol by a mixed microbial culture isolated from paper mill treatment. Gazi Univ. J. Sci. 2014; 27, 839-45.

[19] ZY Hu, GC Du, ZZ Hua and J Chen. Effect of different carbon and nitrogen sources on PVA degradation in a mixed culture and PVA degradation mechanism. J. Food Sci. Biotechnol. 2006; 25, 73-8.

[20] JRA Fried. A Text Book of Polymer Science and Technology. Prentice Hall PTR, New Jersey, USA, 1995.

[21] NPGA Roeges. A Text Book of a Guide to the Complete Interpretation of Infrared Spectra of Organic Structures. Wiley \& Sons, Chichester, England, 1994.

[22] X Hu, R Mamoto, Y Shimomura, K Kimbara and F Kawai. Cell surface structure enhancing uptake of polyvinyl alcohol (PVA) is induced by PVA in the PVA-utilizing Sphingopyxis sp. strain 113P3. Arch. Microbiol. 2007; 188, 235-41.

[23] K Momma, M Okamota, Y Mishima, S Mori, W Hashimoto and K Murata. A novel bacterial ATPbinding cassette transporter system that allows uptake of macromolecules. J. Bacteriol. 2000; 18, 3998-4004. 